Bull. Austral. Math. Soc.

VOL. 67 (2003) [413-427]

\title{
UNBOUNDED PRINCIPAL EIGENFUNCTIONS AND THE LOGISTIC EQUATION ON $R^{N}$
}

\author{
Wei Dong AND Yihong DU
}

\begin{abstract}
We consider the logistic equation $-\Delta u=a(x) u-b(x) u^{p}$ on all of $R^{N}$ with possibly unbounded coefficients near infinity. We show that under suitable growth conditions of the coefficients, the behaviour of the positive solutions of the logistic equation can be largely determined. We also show that certain linear eigenvalue problems on all of $R^{N}$ have principal eigenfunctions that become unbounded near infinity at an exponential rate. Using these results, we finally show that the logistic equation has a unique positive solution under suitable growth restrictions for its coefficients.
\end{abstract}

\section{INTRODUCTION}

We consider the logistic elliptic equation

$$
-\Delta u=a(x) u-b(x) u^{p} ; \quad x \in R^{N},
$$

where $p$ is a constant greater than $1, a(x)$ and $b(x)$ are continuous functions with $b(x)$ positive on $R^{N}$. Equations of this kind have attracted extensive study because of its interest to mathematical biology and Riemannian geometry. We refer to $[\mathbf{2}, \mathbf{5}, \mathbf{8}, \mathbf{9}, \mathbf{1 2}]$ and the references therein for some of the previous research.

When the limits

$$
a_{\infty}=\lim _{|x| \rightarrow \infty} a(x) \text { and } \quad b_{\infty}=\lim _{|x| \rightarrow \infty} b(x)
$$

exist and are positive numbers, it is shown in [8] that problem (1.1) has a unique positive solution $u$, and moreover

$$
u(x) \rightarrow\left(a_{\infty} / b_{\infty}\right)^{1 /(p-1)} \text { as }|x| \rightarrow \infty .
$$

In this paper, we consider cases where these limits may not exist. We suppose that for some $\gamma \geqslant 0$, there exist positive numbers $\alpha_{1}, \alpha_{2}$ and $\beta_{1}, \beta_{2}$ such that

$$
\alpha_{1}=\varliminf_{|x| \rightarrow \infty} \frac{a(x)}{|x|^{\gamma}}, \alpha_{2}=\varlimsup_{|x| \rightarrow \infty} \frac{a(x)}{|x|^{\gamma}}, \beta_{1}=\varliminf_{|x| \rightarrow \infty} b(x), \beta_{2}=\varlimsup_{|x| \rightarrow \infty} b(x) .
$$

It is easily shown that under these conditions, (1.1) has at least one (weak) positive solution (see Corollary 3.3 in Section 3). By standard regularity theory of elliptic equations, any $W_{\text {loc }}^{1,2}\left(R^{N}\right)$ solution of (1.1) belongs to $C^{\mathbf{l}}\left(R^{N}\right)$.

Received 23rd July, 2002

Copyright Clearance Centre, Inc. Serial-fee code: 0004-9727/03 \$A2.00+0.00. 
TheOREM 1.1. Suppose $u \in C^{1}\left(R^{N}\right)$ is a positive solution of (1.1). If (1.2) is satisfied, then

$$
\varliminf_{|x| \rightarrow \infty} \frac{u(x)}{|x|^{\gamma /(p-1)}} \geqslant\left(\frac{\alpha_{1}}{\beta_{2}}\right)^{1 /(p-1)}, \varlimsup_{|x| \rightarrow \infty} \frac{u(x)}{|x|^{\gamma /(p-1)}} \leqslant\left(\frac{\alpha_{2}}{\beta_{1}}\right)^{1 /(p-1)} .
$$

If in addition, we suppose that

$$
p \frac{\alpha_{1} \beta_{1}}{\alpha_{2} \beta_{2}}>1
$$

then (1.1) has a unique positive solution, namely

THEOREM 1.2. Suppose that the conditions (1.2) and (1.3) are satisfied. Then (1.1) has a unique positive solution.

These results considerably improve the corresponding ones in [8]. For the proofs, apart from further development of the techniques used in [8], one main new ingredient is the use of certain unbounded principal eigenfunctions for problems of the type

$$
\Delta \phi=\lambda \xi(x) \phi, \quad x \in R^{N},
$$

where $\xi(x)$ is sign-changing but is positive and bounded away from zero for large $|x|$. The existence of such eigenfunctions will be discussed in detail in Section 2 below. The proofs for Theorems 1.1 and 1.2 will be carried out in Section 3. Some of the ideas in this paper were motivated by $[\mathbf{1}, \mathbf{5}]$ and $[\mathbf{1 2}]$. therein.

For discussions of (1.1) with $\varliminf_{|x| \rightarrow \infty} a(x) \leqslant 0$, we refer to $[2,9]$ and the references

\section{UNBOUNDED PRINCIPAL EIGENFUNCTIONS}

The main purpose of this section is to show that certain eigenfunctions $\phi \in W_{\mathrm{loc}}^{1,2}\left(R^{N}\right)$ of $\Delta \phi=\lambda \xi(x) \phi$ with $\xi(x)$ sign-changing but positive and bounded away from zero for large $|x|$, must become unbounded at an exponential rate as $|x| \rightarrow \infty$. Apart from its own interest, this is crucial for our analysis of the solutions to the logistic equation.

We consider the following eigenvalue problem on $R^{N}$,

$$
\Delta \phi=\lambda \xi(x) \phi, \quad x \in R^{N},
$$

where $\xi(x)$ satisfies

$$
\left\{\begin{array}{l}
\xi \in C\left(R^{N}\right), \xi\left(x_{0}\right)<0 \text { for some } x_{0} \in R^{N} \\
\xi(x) \geqslant \delta>0 \quad \forall|x| \geqslant R>0 \text { for some constant } \delta>0 \text { and large } R .
\end{array}\right.
$$

It is well known (see [6, Theorem 2.1]) that under condition (2.2), the eigenvalue problem (2.1) has a positive principal eigenvalue $\lambda^{*}$ corresponding to which there is a 
positive principal eigenfunction $\phi(x)$ satisfying $\phi(x) \rightarrow 0$ as $|x| \rightarrow \infty$, where $\lambda^{*}$ is given by

$$
\lambda^{*}=\inf \left\{\int_{R^{N}}|\nabla u|^{2} d x / \int_{R^{N}}(-\xi) u^{2} d x: u \in H^{1}\left(R^{N}\right), \int_{R^{N}} \xi u^{2} d x<0\right\} .
$$

Furthermore, a recent result in [1] implies that under these conditions, for any $\lambda \in\left(0, \lambda^{*}\right),(2.1)$ has a positive solution and none of which tend to zero as $|x| \rightarrow \infty$, at least when $N \geqslant 3$. It should be noted that the results in [1] are proved under much weaker assumptions than (2.2).

In the following theorem, we show that in the radial case and under (2.2), much more can be said. For the general case, a weaker result can be obtained as an easy consequence of this theorem.

Theorem 2.1. Suppose that $\xi(x)$ satisfies condition (2.2) and is radially symmetric. Then, for any $\lambda \in\left(0, \lambda^{*}\right)$, there exists a radially symmetric positive function $\phi \in C^{2}\left(R^{N}\right)$ solving (2.1) and such that $\phi(x) \rightarrow \infty$ as $|x| \rightarrow \infty$. Furthermore, for any $q \in(0, \sqrt{\lambda \delta})$, there exist positive constants $R_{*}=R_{*}(q)$ and $C=C(q)$ such that

$$
\phi(x) \geqslant C e^{q|x|} \quad \text { for }|x| \geqslant R_{*} .
$$

Proof: By [1] and [6], we know that equation (2.1) has a positive solution for $\lambda \in\left[0, \lambda^{*}\right]$ with $\lambda^{*}>0$. From the proof of $\left[1\right.$, Theorem 2.3] (but we use the $L^{p}$-theory instead of the Holder theory), we see that when $\lambda \in\left(0, \lambda^{*}\right),(2.1)$ has a positive solution $\phi \in C^{1}\left(R^{N}\right)$ which is the limit of a sequence of radially symmetric functions $\phi_{n}$ satisfying $\Delta \phi_{n}=\lambda \xi(|x|) \phi_{n}, \phi_{n}(x)>0$ for $|x|<n$, and $\phi_{n}(0)=1$. It follows that $\phi$ is radially symmetric.

Denote $\phi(r)=\phi(x)$, where $r=|x|$. We find that $\phi(r)$ must be $C^{2}$ and is a solution of the ODE

$$
\phi^{\prime \prime}(r)+\frac{N-1}{r} \phi^{\prime}(r)=\lambda \xi(r) \phi(r)
$$

satisfying $\phi(0)=1$ and $\phi^{\prime}(0)=0$.

We claim that, for $r>R, \phi$ can not have a local maximum. Indeed, if $\phi\left(r_{0}\right)$ is a local maximum with $r_{0}>R$, then

$$
\phi^{\prime}\left(r_{0}\right)=0, \phi^{\prime \prime}\left(r_{0}\right) \leqslant 0 \text { and } 0 \geqslant \phi^{\prime \prime}\left(r_{0}\right)+\frac{N-1}{r_{0}} \phi^{\prime}\left(r_{0}\right)=\lambda \xi\left(r_{0}\right) \phi\left(r_{0}\right) .
$$

This is a contradiction. This fact implies that for some $R_{1}>R, \phi(r)$ is monotone increasing or decreasing in $\left[R_{1}, \infty\right)$.

We claim that $\phi$ is increasing for $r \geqslant R_{1}$. Otherwise, $\phi$ is decreasing for $r \geqslant R_{1}$, and we must have

$$
\lim _{r \rightarrow \infty} \phi(r)=\eta \geqslant 0, \phi(r) \geqslant \eta, \forall r \geqslant R_{1}
$$


There are two possibilities.

(i) If $\eta>0$, then for $r \geqslant R_{1}$, we have $\phi(r) \geqslant \eta$, and

$$
\left[r^{N-1} \phi^{\prime}(r)\right]^{\prime}=\lambda r^{N-1} \xi(r) \phi(r) \geqslant \lambda r^{N-1} \delta \eta, \quad \forall r \geqslant R_{1} .
$$

Integrating (2.4) over $\left[R_{1}, r\right]$, we obtain

$$
r^{N-1} \phi^{\prime}(r) \geqslant R_{1}^{N-1} \phi^{\prime}\left(R_{1}\right)+\frac{\lambda \delta \eta}{N} r^{N}-\frac{\lambda \delta \eta}{N} R_{1}^{N} .
$$

So

$$
\phi^{\prime}(r) \geqslant \frac{\lambda \delta \eta}{N} r+\left(\frac{R_{1}}{r}\right)^{N-1} \phi^{\prime}\left(R_{1}\right)-\lambda \delta \eta R_{1}\left(\frac{R_{1}}{r}\right)^{N-1}
$$

If $r$ is large enough, the right-hand side of $(2.5)$ is positive, a contradiction to $\phi^{\prime}(r) \leqslant 0$.

(ii) If $\eta=0$, due to (2.2), in the case $N \geqslant 3$, we can use [1, Theorem 1.2] to conclude that no positive solution of (2.1) tends to zero as $|x| \rightarrow \infty$. This is a contradiction to $\eta=0$. Therefore the case $\eta=0$ cannot occur. In the following, we give a unified proof for this fact covering all cases $N \geqslant 1$. Note, however, our condition (2.2) is much more restrictive than that used in $[\mathbf{1}]$.

From (2.3), we obtain

$$
\left[r^{N-1} \phi^{\prime}(r)\right]^{\prime}=\lambda r^{N-1} \xi(r) \phi(r) .
$$

Integrating (2.6) over $\left[R_{1}, r\right]$, we obtain

$$
r^{N-1} \phi^{\prime}(r)-R_{1}^{N-1} \phi^{\prime}\left(R_{1}\right)=\lambda \int_{R_{1}}^{r} s^{N-1} \xi(s) \phi(s) d s .
$$

Since $\phi^{\prime}(r) \leqslant 0$ for $r \geqslant R_{1}$, it follows

$$
\lambda \int_{R_{1}}^{\tau} s^{N-1} \xi(s) \phi(s) d s \leqslant-R_{1}^{N-1} \phi^{\prime}\left(R_{1}\right), \forall r>R_{1} .
$$

This implies that

$$
\int_{R_{1}}^{\infty} s^{N-1} \xi(s) \phi(s) d s<\infty
$$

Therefore, since $\phi \in L^{\infty}([0, \infty))$ and $\xi(r) \geqslant \delta>0$ for $r>R_{1}$, we can conclude that

$$
\int_{0}^{\infty} r^{N-1} \phi^{2}(r) d r<\infty, \int_{0}^{\infty} r^{N-1} \xi(r) \phi^{2}(r) d r<\infty
$$

We now multiply (2.6) by $\phi(r)$ and integrate over $[0, r]$ to deduce

$$
r^{N-1} \phi^{\prime}(r) \phi(r)-\int_{0}^{r} s^{N-1} \phi^{\prime}(s)^{2} d r=\lambda \int_{0}^{r} s^{N-1} \xi(s) \phi^{2}(s) d s .
$$


By (2.7) and (2.8), we find that $r^{N-1} \phi^{\prime}(r)$ has a finite limit as $r \rightarrow \infty$. By assumption, $\phi(r) \rightarrow \eta=0$ as $r \rightarrow \infty$. Therefore

$$
r^{N-1} \phi^{\prime}(r) \phi(r) \rightarrow 0 \text { as } r \rightarrow \infty .
$$

Using this fact and letting $r \rightarrow \infty$ in (2.10), noticing also (2.9), we find that

$$
\int_{0}^{\infty} s^{N-1} \phi^{\prime}(s)^{2} d s<\infty, \int_{0}^{\infty} s^{N-1} \phi^{\prime}(s)^{2} d x=-\lambda \int_{0}^{\infty} s^{N-1} \xi(s) \phi^{2}(s) d s .
$$

Let us note that the first identities in (2.9) and (2.11) imply that $\phi \in H^{1}\left(R^{N}\right)$, while the second identity in (2.11) is equivalent to

$$
\lambda=\int_{R^{N}}|\nabla \phi|^{2} d x / \int_{R^{N}}(-\xi) \phi^{2} d x
$$

But by the definition of $\lambda^{*}$, we have

$$
\lambda^{*} \leqslant \int_{R^{N}}|\nabla \phi|^{2} d x / \int_{R^{N}}(-\xi) \phi^{2} d x
$$

Thus we arrive at the contradiction $\lambda^{*} \leqslant \lambda$.

Summarising, we arrive at a contradiction in each case when assuming $\phi(r)$ is decreasing for $r>R_{1}$. This proves our claim that $\phi(r)$ is increasing for $r>R_{1}$.

Next we shall show $\phi(r) \geqslant C e^{q r}$ for all large $r$. By a simple calculation we find that $u(r):=r^{(N-1) / 2} \phi(r)$ satisfies

$$
u^{\prime \prime}-q_{1}(r) u=0
$$

where

$$
q_{1}(r)=\lambda \xi(r)+\frac{(N-1)(N-3)}{4 r^{2}} .
$$

By condition (2.2), for any $\delta_{1} \in(0, \delta)$, we can find $R_{2} \geqslant R_{1}$ such that

$$
q_{1}(r)>\lambda \delta_{1}=: \delta_{0}^{2}, \forall r \geqslant R_{2} .
$$

Let $v(r)=e^{\delta_{0}\left(r-R_{2}\right)}+e^{-\delta_{0}\left(r-R_{2}\right)}$. Clearly $v$ satisfies

$$
v^{\prime \prime}-\delta_{0}^{2} v=0, v>0, \forall r \in(-\infty, \infty), v^{\prime}\left(R_{2}\right)=0 .
$$

Multiplying (2.12) by $v$ and (2.13) by $u$, subtracting and integrating over $\left[R_{2}, r\right]$, we obtain

$$
u^{\prime}(r) v(r)-u(r) v^{\prime}(r) \geqslant u^{\prime}\left(R_{2}\right) v\left(R_{2}\right)-u\left(R_{2}\right) v^{\prime}\left(R_{2}\right)=2 u^{\prime}\left(R_{2}\right) \geqslant 0 .
$$

It follows $(u / v)^{\prime} \geqslant 0$, and hence

$$
\frac{u}{v} \geqslant \frac{u\left(R_{2}\right)}{v\left(R_{2}\right)}=: a_{0}>0, \forall r \geqslant R_{2}
$$


Therefore,

$$
\phi(r)=r^{(1-N) / 2} u(r) \geqslant a_{0} r^{(1-N) / 2} e^{\delta_{0}\left(r-R_{2}\right)} .
$$

Since $\delta_{1} \in(0, \delta)$ is arbitrary, for any given $q \in(0, \sqrt{\lambda \delta})$, we may assume that $\delta_{1}$ is chosen so that $\delta_{0}=\sqrt{\lambda \delta_{1}}>q$. We now see from (2.14) that for some positive constants $C=C(q)$ and $R_{*}=R_{*}(q)$,

$$
\phi(r) \geqslant C e^{q r}, \forall r>R_{*}
$$

This finishes the proof of the theorem.

Corollary 2.2. Suppose that $\xi(x)$ satisfies (2.2) and for $r \geqslant 0$ let $\bar{\xi}(r)$ $=\min _{|x|=r} \xi(x)$. Then

$$
\vec{\lambda}^{*}:=\inf \left\{\int_{R^{N}}|\nabla u|^{2} d x / \int_{R^{N}}(-\bar{\xi}) u^{2} d x: u \in H^{1}\left(R^{N}\right), \int_{R^{N}} \bar{\xi} u^{2} d x<0\right\}
$$

is positive, and for each $\lambda \in\left(0, \bar{\lambda}^{*}\right)$, there exists a positive function $\phi \in C^{1}\left(R^{N}\right)$ solving (2.1). Moreover, for any such $\phi$ and any $q \in(0, \sqrt{\lambda \delta})$, there exist positive constants $R_{*}$ and $C$ such that

$$
\max _{|x|=r} \phi(x) \geqslant C e^{q r} \quad \text { for } r \geqslant R_{*} .
$$

Proof: Since $\bar{\xi} \leqslant \xi$, from the definition of the principal eigenvalue $\lambda^{*}$ for (2.1), we find $\lambda^{*} \geqslant \vec{\lambda}^{*}$. Therefore, for each $\lambda \in\left(0, \vec{\lambda}^{*}\right)$, by the method in [1], (2.1) has a positive solution $\phi \in C^{1}\left(R^{N}\right)$.

To show (2.15), we argue indirectly. Suppose that for some $\lambda \in\left(0, \vec{\lambda}^{*}\right)$ and some $q \in(0, \sqrt{\lambda \delta}),(2.1)$ has a positive solution $\phi$ satisfying

$$
\varliminf_{r \rightarrow \infty} \max _{|x|=r} \phi(x) / e^{g r}=0 .
$$

By definition, $\bar{\xi}$ satisfies (2.2) with the same $\delta$. By Theorem 2.1, we can find a radially symmetric positive function $\bar{\phi}$ such that

$$
\Delta \bar{\phi}=\lambda \overline{\xi \phi}, \bar{\phi}>0 \text { in } R^{N}
$$

and

$$
\bar{\phi}(|x|) \geqslant C e^{q|x|} \text { for some } C>0 \text { and all large }|x| \text {. }
$$

Denote

$$
a_{r}=\max _{|x| \leqslant r} \phi(x) / \bar{\phi}(|x|) .
$$

Clearly $a_{r}>0$ and due to (2.16), (2.17), there is a sequence $\left\{r_{n}\right\}$ satisfying $r_{n} \rightarrow \infty$ such that $a_{r_{n}}$ is achieved at some $x_{n}$ with $\left|x_{n}\right|<r_{n}$. Then $\psi_{n}(x):=a_{r_{n}} \bar{\phi}(|x|)-\phi(x)$ is nonnegative in $B_{r_{n}}$, it vanishes at $x_{n}$ and is positive for $|x|=r_{n}$. 
It is easily checked that, in the weak sense,

$$
\Delta \psi_{n} \leqslant \lambda \xi \psi_{n} \text { in } B_{r_{n}} .
$$

Let $u_{n}=\psi_{n} / \phi$. Since $\phi$ satisfies

$$
\Delta \phi=\lambda \xi \phi, \phi>0 \text { in } R^{N},
$$

a simple calculation yields

$$
\Delta u_{n}+2 \phi^{-1} \nabla \phi \cdot \nabla u_{n} \leqslant 0 \text { in } B_{r_{n}} .
$$

Therefore due to $u_{n}=\psi_{n} / \phi \geqslant 0$ and $u_{n}\left(x_{n}\right)=\psi_{n}\left(x_{n}\right) / \phi\left(x_{n}\right)=0$, one can apply the strong maximum principle (see $\left[10\right.$, Theorem 9.6]) to conclude that $u_{n} \equiv 0$ on $B_{r_{n}}$, a contradiction. This finishes the proof.

\section{Proof of the MAIN RESUlts}

In this section, we first prove the asymptotic behaviour of the positive solutions of (1.1) as given in Theorem 1.1, and then make use of this result and Theorem 2.1 in Section 2 to prove the uniqueness result in Theorem 1.2.

To start, we recall a comparison principle (see, for example, [8, Lemma 2.1]) which will be frequently used in the later proof.

Lemma 3.1. (Comparison principle) Suppose that $\Omega$ is a bounded domain in $R^{N}$. Let $u_{1}, u_{2} \in C^{1}(\Omega)$ be positive in $\Omega$ and satisfy (in the weak sense)

$$
\Delta u_{1}+a(x) u_{1}-b(x) u_{1}^{p} \leqslant 0 \leqslant \Delta u_{2}+a(x) u_{2}-b(x) u_{2}^{p} \quad \text { in } \Omega
$$

and

$$
\varlimsup_{d(x, \partial \Omega) \rightarrow 0}\left(u_{2}-u_{1}\right) \leqslant 0 .
$$

where $p>1, a(x), b(x)$ are continuous with $b(x)$ positive on $\Omega$ and $\|a\|_{L^{\infty}(\Omega)}<\infty$. Then $u_{2} \leqslant u_{1}$ in $\Omega$.

It should be noted that in Lemma 3.1, the assumption that $u_{1}$ and $u_{2}$ are positive and satisfy (3.1) in $\Omega$ has hidden restrictions on $a(x)$ and $b(x)$. Moreover, from the proof in $[8]$ one easily sees that the restriction that $u_{1}, u_{2} \in C^{2}(\Omega)$ there can be replaced by $u_{1}, u_{2} \in C^{1}(\Omega)$.

Next we present an existence result which is folklore. As we cannot find it in the literature, for completeness, a proof is provided.

PROPOSITION 3.2. If $\lambda_{1}\left(B_{r}\right)<0$ for some $r>0$, then (1.1) possess a minimal positive solution $\underline{u}$ and a maximal positive solution $\bar{u}$, where $\lambda_{1}\left(B_{r}\right)$ denotes the first eigenvalue of the problem

$$
-\Delta u-a(x) u=\lambda u,\left.\quad u\right|_{\partial B_{r}}=0,
$$

and $B_{r}$ is the ball centered at the origin with radius $r$. 
Proof: By the properties of the first eigenvalue (see, for example, [4]), for all $R \geqslant r$, $\lambda_{1}\left(B_{R}\right)<0$. Let $\phi$ be a positive eigenfunction corresponding to $\lambda_{1}\left(B_{R}\right)$. Then for all small positive constant $\varepsilon$, it is easily checked that $\varepsilon \phi$ is a lower solution of the problem

$$
-\Delta u=a(x) u-b(x) u^{p},\left.\quad u\right|_{\partial B_{R}}=0
$$

Clearly any positive constant greater than or equal to $\bar{M}=\max _{B_{R}}(a(x) / b(x))^{1 /(p-1)}$ is an upper solution of (3.3). Thus (3.3) has at least one positive solution. By Lemma 3.1, it has a unique positive solution.

Let us choose an increasing sequence of positive real numbers $r_{n}$ with $r_{1}>r$ and $r_{n} \rightarrow \infty$ as $n \rightarrow \infty$. By the properties of the first eigenvalue in [4], and by the above discussion, problem (3.3) with $r=r_{n}$ has a unique positive solution $u_{n}$. By Lemma 3.1, we deduce $u_{n} \leqslant u_{n+1}$. If we can find an upper bound for $u_{n}$ on any fixed $B_{R}$, then by a standard regularity argument, $\underline{u}(x)=\lim _{n \rightarrow \infty} u_{n}(x)$ is well-defined in $R^{N}$ and it would be a positive solution of (1.1). To find such an upper bound, we consider the problem

$$
-\Delta v=a(x) v-b(x) v^{p},\left.\quad v\right|_{\partial B_{R}}=\infty .
$$

(Here and throughout this paper, by $\left.v\right|_{\partial B_{R}}=\infty$, we mean $v \rightarrow \infty$ as $\operatorname{dist}\left(x, \partial B_{R}\right) \rightarrow 0$.) By [3] (see also [11] and [7]), (3.4) has a positive solution $v$. Then clearly by Lemma 3.1,

$$
u_{n}(x) \leqslant v(x), \forall x \in B_{R}
$$

for all large $n$ so that $r_{n}>R$. This is the bound we are looking for, and hence the existence of a solution for (1.1) is proved.

From $u_{n} \leqslant u_{n+1}$ we find

$$
\underline{u}(x) \geqslant u_{n}(x)>0
$$

for each $n$, and hence $\underline{u}$ is a positive solution of (1.1). For an arbitrary positive solution $u$ of (1.1), we can see that $u$ satisfies

$$
-\Delta u=a(x) u-b(x) u^{p},\left.\quad u\right|_{\partial B_{r_{n}}}>0
$$

By Lemma 3.1, $u \geqslant u_{n}$ on $B_{r_{n}}$ for each $n$, and hence

$$
u \geqslant \underline{u}=\lim _{n \rightarrow \infty} u_{n}
$$

So $\underline{u}$ is the minimal positive solution of (1.1).

What remains now is to show the existence of a maximal positive solution of (1.1). To this end, we choose an increasing sequence of real number $r_{n}$ such that $r_{n} \rightarrow \infty$ as $n \rightarrow \infty$ and denote $B_{n}=B_{r_{n}}$. Next we consider the boundary blow-up problem

$$
-\Delta \omega=a(x) \omega-b(x) \omega^{p},\left.\quad \omega\right|_{\partial B_{n}}=\infty
$$


By [3] (see also [11] and [7]), (3.5) has a unique positive solution which we denote as $\omega_{n}$. Applying Lemma 3.1 , we see $\omega_{n} \geqslant \omega_{n+1} \geqslant \underline{u}$ for all $n$, so

$$
\bar{u}=\lim _{n \rightarrow \infty} \omega_{n}
$$

is well-defined on $R^{N}$. Furthermore, by standard regularity considerations, we know $\bar{u}$ satisfies (1.1) on $R^{N}$ and $\bar{u} \geqslant \underline{u}$, so $\bar{u}$ is a positive solution of (1.1).

Clearly any positive solution $u$ of (1.1) satisfies, for each $n$,

$$
-\Delta u=a(x) u-b(x) u^{p},\left.\quad u\right|_{\partial B_{n}}<\infty
$$

By Lemma 3.1, we obtain $\omega_{n} \geqslant u$ on $B_{n}$ for all $n$, and hence

$$
\bar{u}=\lim _{n \rightarrow \infty} \omega_{n} \geqslant u
$$

The proof is now complete.

When condition (1.2) is satisfied, it is easily seen that $\lambda_{1}\left(B_{r}\right)<0$ for all large $r$. Therefore, we have the following result.

Corollary 3.3. Under condition (1.2), problem (1.1) has a minimal positive solution and a maximal positive solution.

We are now ready to prove Theorem 1.1. We consider the cases $\gamma=0$ and $\gamma>0$ separately, as the proofs we use are considerably different.

LEMMA 3.4. Assume that $u \in C^{1}\left(R^{N}\right)$ is a positive solution of (1.1). If condition (1.2) is satisfied with $\gamma=0$, then

$$
\varliminf_{|x| \rightarrow \infty} u^{p-1}(x) \geqslant \frac{\alpha_{1}}{\beta_{2}}
$$

and

$$
\varlimsup_{|x| \rightarrow \infty} u^{p-1}(x) \leqslant \frac{\alpha_{2}}{\beta_{1}}
$$

Proof: For any small positive $\varepsilon$ such that $3 \varepsilon<\min \left\{\alpha_{1}, \beta_{1}\right\}$, there exists a large $R$ such that

$$
|x|>R \text { implies } a(x) \geqslant a_{0}:=\alpha_{1}-\varepsilon, \quad b(x) \leqslant b_{0}:=\beta_{2}+\varepsilon
$$

Set

$$
\bar{a}(x)=\psi(x) a(x)+(1-\psi(x)) a_{0}, \quad \bar{b}(x)=\psi(x) b(x)+(1-\psi(x)) b_{0},
$$

where $\psi(x)$ is a smooth cutoff function such that

$$
\left\{\begin{array}{l}
\psi(x) \equiv 1, \quad \forall|x| \leqslant R, \quad \psi(x) \equiv 0, \quad \forall|x| \geqslant 2 R \\
0 \leqslant \psi(x) \leqslant 1, \quad \forall|x| \in[R, 2 R]
\end{array}\right.
$$


Let us now choose an increasing sequence of real numbers $r_{n}$ with $r_{1}>R$ large enough such that $\lambda_{1}\left(B_{r_{1}}\right)<0$. As in the proof of Proposition 3.2, the problem

$$
-\Delta v=\bar{a}(x) v-\bar{b}(x) v^{p},\left.\quad v\right|_{\partial B_{r_{n}}}=0
$$

has a unique positive solution $v_{n}$. Then $v(x)=\lim _{|x| \rightarrow \infty} v_{n}(x)$ is well-defined on $R^{N}$, and by standard regularity considerations $v(x)$ is a positive solution of the problem

$$
-\Delta v=\bar{a}(x) v-\bar{b}(x) v^{p}, \quad x \in R^{N} .
$$

Since $\lim _{|x| \rightarrow \infty} \bar{a}(x)=a_{0}>0$ and $\lim _{|x| \rightarrow \infty} \vec{b}(x)=b_{0}>0$, by Theorem 3.1 in [8], $v(x)$ is the unique positive solution of (3.10) and

$$
\lim _{|x| \rightarrow \infty} v(x)=\left(\frac{a_{0}}{b_{0}}\right)^{1 /(p-1)} .
$$

On the other hand, any positive solution $u(x)$ of (1.1) satisfies

$$
-\Delta u=a(x) u-b(x) u^{p} \geqslant \bar{a}(x) u-\bar{b}(x) u^{p},\left.\quad u\right|_{\partial B_{r}}>0 .
$$

So by Lemma 3.1, $u(x) \geqslant v_{n}(x), \quad \forall x \in B_{r_{n}}$, and hence

$$
u(x) \geqslant v(x), \forall x \in R^{N}, \varliminf_{|x| \rightarrow \infty} u(x) \geqslant \lim _{|x| \rightarrow \infty} v(x)=\left(a_{0} / b_{0}\right)^{1 /(p-1)} .
$$

Therefore (3.6) holds due to the arbitrariness of $\varepsilon$.

It remains to show (3.7). By (1.2), for any small positive $\varepsilon$, there exists a large $R$ such that

$$
a(x) \leqslant \alpha_{2}+\varepsilon=: a_{\infty}, \quad b(x) \geqslant \beta_{1}-\varepsilon=: b_{\infty}, \forall|x| \geqslant R
$$

Set

$$
\bar{a}(x)=a(x) \psi(x)+(1-\psi(x)) a_{\infty}, \quad \bar{b}(x)=b(x) \psi(x)+(1-\psi(x)) b_{\infty},
$$

where $\psi(x)$ is a cutoff function as in (3.8). Let us now consider the problem

$$
-\Delta \omega=\bar{a}(x) \omega-\bar{b}(x) \omega^{p},\left.\quad \omega\right|_{\partial B_{r}}=\infty
$$

By [3] (see also [11] and [7]), this problem has a unique positive solution $\omega_{r}, \omega=\lim _{r \rightarrow \infty} \omega_{r}$ is well-defined on $R^{N}$ and is a positive solution of the equation

$$
-\Delta \omega=\bar{a}(x) \omega-\bar{b}(x) \omega^{p}, \quad x \in R^{N} .
$$

Since $\lim _{|x| \rightarrow \infty} \bar{a}(x)=a_{\infty}>0$ and $\lim _{|x| \rightarrow \infty} \bar{b}(x)=b_{\infty}>0$, by Theorem 3.1 in [8], $\omega(x)$ is the unique positive solution of (3.12) and $\lim _{|x| \rightarrow \infty} \omega(x)=\left(a_{\infty} / b_{\infty}\right)^{1 / p-1}$. On the other hand, if $u$ is a positive solution of (1.1), then

$$
-\Delta u=a(x) u-b(x) u^{p} \leqslant \bar{a}(x) u-\bar{b}(x) u^{p},\left.u\right|_{\partial B_{r}}<\infty .
$$


By Lemma 3.1, we obtain $u(x) \leqslant \omega_{r}(x), \forall x \in B_{r}$, and thus

$$
\varlimsup_{|x| \rightarrow \infty} u \leqslant \lim _{|x| \rightarrow \infty} \omega=\left(\frac{a_{\infty}}{b_{\infty}}\right)^{1 /(p-1)} .
$$

Since $\varepsilon$ is arbitrary, (3.7) follows.

Lemma 3.5. Suppose $u \in C^{1}\left(R^{N}\right)$ is a positive solution of (1.1). If (1.2) is satisfied with $\gamma>0$, then

$$
\varliminf_{|x| \rightarrow \infty} \frac{u^{p-1}(x)}{|x|^{\gamma}} \geqslant \frac{\alpha_{1}}{\beta_{2}}
$$

and

$$
\varlimsup_{|x| \rightarrow \infty} \frac{u^{p-1}(x)}{|x|^{\gamma}} \leqslant \frac{\alpha_{2}}{\beta_{1}}
$$

Proof: Let $\left\{x_{n}\right\}$ be an arbitrary sequence of points $x_{n}$ in $R^{N}$ satisfying $r_{n}=\left|x_{n}\right|$ $\rightarrow \infty$ and let $\varepsilon>0$ be such that $3 \varepsilon<\min \left\{\alpha_{1}, \beta_{1}\right\}$. By (1.2), there exists a large $R>0$ such that

$$
a(x) \geqslant a_{0}|x|^{\gamma}, \quad b(x) \leqslant b_{0}, \quad \forall|x|>R,
$$

where $a_{0}=\alpha_{1}-\varepsilon, \quad b_{0}=\beta_{2}+\varepsilon$.

For any fixed $x_{0} \in R^{N}$, let

$$
B_{0}=\left\{x: R_{1}<|x|<R_{2}\right\}, \text { where } R_{1}=(1-\varepsilon)\left|x_{0}\right|, \quad R_{2}=(1+\varepsilon)\left|x_{0}\right| .
$$

We consider the following problem

$$
-\Delta v=a_{0} R_{1}^{\gamma} v-b_{0} v^{p},\left.\quad v\right|_{\partial B_{0}}=0 .
$$

If $v_{0}(x)$ is a positive solution of (3.15), then by Lemma 3.1 it must be the unique positive solution, and hence it must be radially symmetric: $v_{0}(x)=v_{0}(|x|)$. Let

$$
v_{0}(r)=R_{1}^{\gamma /(p-1)} \omega(r)
$$

Then

$$
-\Delta \omega=R_{1}{ }^{\gamma} \omega\left(a_{0}-b_{0} \omega^{(p-1)}\right),\left.\quad \omega\right|_{\partial B_{0}}=0 .
$$

Through a simple rescaling $\omega(r)=z\left(r / R_{1}\right)$, we obtain

$$
-\Delta z=R_{1}^{\gamma+2} z\left(a_{0}-b_{0} z^{p-1}\right),\left.\quad z\right|_{\partial \Omega}=0,
$$

where $\Omega=\{x: 1<|x|<(1+\varepsilon) /(1-\varepsilon)\}$. By $\left[8\right.$, Lemma 2.2], for all large $R_{1},(3.16)$ has a unique positive solution $z(x)$ and $z(x) \rightarrow\left(a_{0} / b_{0}\right)^{1 /(p-1)}$ uniformly on any compact 
subset of $\Omega$ as $R_{1} \rightarrow \infty$. Therefore (3.15) does have a unique positive solution $v_{0}(x)$ as described above, and there exists a large $R_{*}>R$ such that for $R_{1}>R_{*}$,

$$
v_{0}^{p-1}\left(x_{0}\right) \geqslant(1-\varepsilon) \frac{a_{0}}{b_{0}} R_{1}^{\gamma} .
$$

It follows that

$$
\frac{v_{0}^{p-1}\left(x_{0}\right)}{\left|x_{0}\right|^{\gamma}} \geqslant(1-\varepsilon)^{\gamma+1} \frac{a_{0}}{b_{0}}
$$

Since $\left|x_{n}\right|=r_{n} \rightarrow \infty$, there exists a positive integer $n_{0}$ such that for $n>n_{0}$, when $x_{0}$ is replaced by $x_{n}$, equation (3.15) has a unique positive solution $v_{n}$. Then by (3.17),

$$
\frac{v_{n}^{p-1}\left(x_{n}\right)}{\left|x_{n}\right|^{\gamma}} \geqslant(1-\varepsilon)^{\gamma+1} \frac{a_{0}}{b_{0}} \text {. }
$$

If $u$ is a positive solution of (1.1), then for all large $n$,

$$
-\Delta u \geqslant a_{0} R_{1}^{\gamma} u-b_{0} u^{p},\left.\quad u\right|_{\partial B_{n}}>0
$$

where

$$
B_{n}=\left\{x:(1-\varepsilon)\left|x_{n}\right|<|x|<(1+\varepsilon)\left|x_{n}\right|\right\} .
$$

By Lemma 3.1, we obtain $v_{n}(x) \leqslant u(x)$ on $B_{n}$. Thus, for all large $n, u\left(x_{n}\right) \geqslant v_{n}\left(x_{n}\right)$, and

$$
\frac{u^{p-1}\left(x_{n}\right)}{\left|x_{n}\right|^{\gamma}} \geqslant \frac{v_{n}^{p-1}\left(x_{n}\right)}{\left|x_{n}\right|^{\gamma}} \geqslant(1-\varepsilon)^{\gamma+1} \frac{a_{0}}{b_{0}} .
$$

Since $\left\{x_{n}\right\}$ is an arbitrary sequence with $\left|x_{n}\right| \rightarrow \infty$, this implies that

$$
\varliminf_{|x| \rightarrow \infty} \frac{u^{p-1}(x)}{|x|^{\gamma}} \geqslant(1-\varepsilon)^{\gamma+1} \frac{a_{0}}{b_{0}} .
$$

Inequality (3.13) then follows as $\varepsilon>0$ can be arbitrarily small.

Next we shall prove (3.14). In a similar fashion, for any sequence of points $x_{n}$ in $R^{N}$ satisfying $\left|x_{n}\right| \rightarrow \infty$, and any given small positive $\varepsilon$, there exists a large $R>0$ such that

$$
a(x) \leqslant a_{1}|x|^{\gamma}, \quad b(x) \geqslant b_{1}, \quad \forall|x|>R,
$$

where $a_{1}=\alpha_{2}+\varepsilon, \quad b_{1}=\beta_{1}-\varepsilon$. We consider the problem

$$
-\Delta v=a_{1} R_{2}^{\gamma} v-b_{1} v^{p},\left.\quad v\right|_{\partial B_{0}}=\infty,
$$

where $B_{0}, R_{2}$ are defined as before. $\mathrm{By}[3]$ (see also $[11,7]$ ), problem (3.18) possesses a unique radially symmetric positive solution $v_{0}(x)=v_{0}(|x|)$. Let

$$
v_{0}(r)=R_{2}^{\gamma /(p-1)} \omega(r)
$$


Then $\omega(r)$ satisfies

$$
-\Delta \omega=R_{2}^{\gamma} \omega\left(a_{1}-b_{1} \omega^{p-1}\right),\left.\quad \omega\right|_{\partial B_{0}}=\infty .
$$

Through a rescaling $\omega(r)=z\left(r / R_{2}\right)$, we obtain

$$
-\Delta z=R_{2}^{\gamma+2} z\left(a_{1}-b_{1} z^{p-1}\right),\left.\quad z\right|_{\partial \Omega}=\infty,
$$

where $\Omega=\{x:(1-\varepsilon) /(1+\varepsilon)<|x|<1\}$. By [8, Lemma 2.3], $z(x) \rightarrow\left(a_{1} / b_{1}\right)^{p-1}$ uniformly on any compact subset of $\Omega$ as $R_{2} \rightarrow \infty$. Thus, there is a large $R_{*}>R$ such that for $R_{1}>R_{*}$,

$$
\frac{v_{0}^{p-1}\left(x_{0}\right)}{\left|x_{0}\right|^{\gamma}} \leqslant(1+\varepsilon)^{\gamma+1} \frac{a_{1}}{b_{1}}
$$

Since $\left|x_{n}\right|=r_{n} \rightarrow \infty$, for all large $n$, the unique positive solution $v_{n}$ of (3.18) with $x_{0}$ replaced by $x_{n}$ satisfies

$$
\frac{v_{n}^{p-1}\left(x_{n}\right)}{\left|x_{n}\right|^{\gamma}} \leqslant \frac{a_{1}}{b_{1}}(1+\varepsilon)^{\gamma+1} .
$$

If $u$ is a positive solution of (1.1), then for all large $n$,

$$
-\Delta u \leqslant a_{1} R_{2}^{\gamma} u-b_{1} u^{p},\left.\quad u\right|_{\partial B_{n}}<\infty,
$$

where $B_{n}=\left\{x:(1-\varepsilon)\left|x_{n}\right|<|x|<(1+\varepsilon)\left|x_{n}\right|\right\}$. By Lemma 3.1, we obtain

$$
u(x) \leqslant v_{n}(x), \forall x \in B_{n}
$$

Thus

$$
\frac{u^{p-1}\left(x_{n}\right)}{\left|x_{n}\right|^{\gamma}} \leqslant \frac{v_{n}^{p-1}\left(x_{n}\right)}{\left|x_{n}\right|^{\gamma}} \leqslant \frac{a_{1}}{b_{1}}(1+\varepsilon)^{\gamma+1}
$$

This implies that

$$
\varlimsup_{|x| \rightarrow \infty} \frac{u^{p-1}(x)}{|x|^{\gamma}} \leqslant \frac{a_{1}}{b_{1}}(1+\varepsilon)^{\gamma+1} .
$$

Inequality (3.14) now follows as $\varepsilon>0$ can be arbitrarily small. This completes the proof of the lemma.

Clearly Theorem 1.1 is a direct consequence of Lammas 3.4 and 3.5. To prove Theorem 1.2, we shall need the following result whose proof uses Theorem 2.1.

LEMma 3.6. Under conditions (1.2) and (1.3), there exists a positive function $\phi \in C^{1}\left(R^{N}\right)$ satisfying $\phi(x) \rightarrow \infty$ as $|x| \rightarrow \infty$, such that for any positive $\varepsilon, \underline{u}+\varepsilon \phi$ is an upper solution of (1.1), that is (in the weak sense),

$$
-\Delta(\underline{u}+\varepsilon \phi) \geqslant a(x)(\underline{u}+\varepsilon \phi)-b(x)(\underline{u}+\varepsilon \phi)^{p},
$$

where $\underline{u}$ is the minimal positive solution of (1.1). 
Proof: Under condition (1.2), due to Corollary 3.3 , (1.1) has a minimal positive solution $\underline{u}$. Let

$$
\sigma(x)=p b(x) \underline{u}^{p-1}-a(x) .
$$

By Lemmas 3.4, 3.5, and by (1.2), (1.3), we have

$$
\varliminf_{|x| \rightarrow \infty} \frac{\sigma(x)}{|x|^{\gamma}} \geqslant p \alpha_{1} \beta_{1} / \beta_{2}-\alpha_{2}>0 .
$$

Therefore, there exists a large $R$ such that $\sigma(x) \geqslant \delta$ for all $|x| \geqslant R$ and some positive $\delta$. We can now easily find a radially symmetric function $\xi(x)=\xi(|x|)$ such that it satisfies (2.2) and $\xi(x) \leqslant \sigma(x), \forall x \in R^{N}$. By Theorem 2.1, we obtain a radially symmetric positive function $\bar{\phi} \in C^{2}\left(R^{N}\right)$ solving, for some $\lambda \in\left(0, \lambda^{*}\right) \cap(0,1)$,

$$
\Delta \bar{\phi}=\lambda \xi(x) \bar{\phi}, \quad \forall x \in R^{N},
$$

and

$$
\bar{\phi}(x) \geqslant C e^{q|x|} \text { for some } C>0, q>0 \text { and all large }|x| .
$$

Since $\lambda \leqslant 1$, for $|x|>R$, from $\xi(x)>0$ we deduce

$$
\lambda \xi(x) \leqslant \xi(x) \leqslant \sigma(x), \forall|x|>R .
$$

We now choose $t>0$ large enough such that

$$
[\lambda \xi(x)-\sigma(x)] \bar{\phi}(x)-t(p-1) b(x) \underline{u}^{p}(x)<0, \forall|x| \leqslant R .
$$

Then define

$$
\phi(x)=\bar{\phi}(x)+t \underline{u}(x) .
$$

We have

$$
\Delta \phi-\sigma(x) \phi=[\lambda \xi(x)-\sigma(x)] \bar{\phi}(x)-t(p-1) b(x) \underline{u}^{p}(x) \leqslant 0 \text { in } R^{N} .
$$

We shall see that $\phi(r)$ meets the requirement in this lemma. By $(3.19), \phi(x) \rightarrow \infty$ as $|x| \rightarrow \infty$. For all positive $\varepsilon$, setting $v=\underline{u}+\varepsilon \phi$, then, by (3.20),

$$
-\Delta v-a(x) v+b(x) v^{p} \geqslant \varepsilon\left[-\Delta \phi-a(x) \phi+p b(x) \underline{u}^{p-1} \phi\right]=\varepsilon[-\Delta \phi+\sigma(x) \phi] \geqslant 0 .
$$

Hence $v=\underline{u}+\varepsilon \phi$ is an upper solution of (1.1). The proof is complete.

We are now ready to complete the proof of Theorem 1.2.

Proof of TheOREM 1.2: By Corollary 3.3, problem (1.1) has a minimal positive solution $\underline{u}$ and a maximal positive solution $\bar{u}$. Due to Lemma 3.6, for any positive $\varepsilon$, $\underline{u}+\varepsilon \phi$ is an upper solution of (1.1). By (3.19) and Lemmas 3.4,3.5, for fixed $\varepsilon$ and all large $|x|, \underline{u}+\varepsilon \phi \geqslant \bar{u}$. This implies, by Lemma $3.1, \underline{u}+\varepsilon \phi \geqslant \bar{u}$ on all large balls. Therefore it is true on all of $R^{N}$. Since $\varepsilon>0$ is arbitrary, it follows that $\underline{u} \geqslant \bar{u}$ on $R^{N}$. On the other hand, $\underline{u} \leqslant \bar{u}$ on $R^{N}$. Thus we must have $\underline{u}=\bar{u}$, which implies that (1.1) has a unique positive solution. The proof of Theorem 1.2 is now complete. 


\section{REFERENCES}

[1] G.A. Afrouzi and K.J. Brown, 'Unbounded principal eigenfunctions for problems on all $R^{N}$, Differential Integral Equations 14 (2001), 37-50.

[2] G.A. Afrouzi and K.J. Brown, 'On a diffusive logistic equation', J. Math. Anal. Appl. 225 (1998), 326-339.

[3] C. Bandle and M. Marcus, 'Asymptotic behavior of solutions and their derivatives for semilinear elliptic problems with blow-up on the boundary', Ann. Inst. H. Poincaré Anal. Nonlinéaire 12 (1995), 155-171.

[4] H. Berestycki, L. Nirenberg and S.R.S. Varadhan, 'The principal engenvalue and maximum principle for second-order elliptic operator in general domains', Comm. Pure Appl. Math. 47 (1994), 47-92.

[5] B. Bianchi and M. Rigoli, 'Nonexistence and uniqueness of positive solutions of Yamabe type equations on nonpositively curved manifolds', Trans. Amer. Math. Soc. 349 (1997), 4753-4774.

[6] K. J. Brown, C. Cosner and J. Fleckinger, 'Principal eigenvalues for problems with indefinite function on $R^{N}$, Proc. Amer. Math. Soc. 109 (1990), 147-155.

[7] Y. Du and S. Li, 'Positive solutions with prescribed patterns in some simple semilinear equations', Differential Integral Equations 15 (2002), 805-822.

[8] Y. Du and L. Ma, 'Logistic type equations on $R^{N}$ by a squeezing method involving boundary blow-up solutions', J. London. Math. Soc. (2) 64 (2001), 107-124.

[9] Y. Du and L. Ma, 'Positive solutions of an elliptic partial differential equation on $R^{N}$, J. Math. Anal. Appl. 271 (2002), 409-425.

[10] D. Gilbarg and N.S. Trudinger, Elliptic partial differential equations of second order, (second edition) (Springer-Verlag, Berlin, 1983).

[11] M. Marcus and L. Veron, 'Uniqueness and asymptotic behavior of solutions with boundary blow-up. for a class of nonlinear elliptic equation', Ann. Inst. H. Poincaré Anal. Nonlinéaire 14 (1997), 237-274.

[12] A. Ratto, M. Rigoli and L. Veron, 'Scalar curvature and conformal deformations of noncompact Riemannian manifolds', Math. Z. 225 (1997), 395-426.

School of Mathematics, Statistics and Computer Science

University of New England

Armidale, NSW 2351

Australia

e-mail: wdong@turing.une.edu.au

ydu@turing.une.edu.au 